Koichi Miyaki $\cdot$ Mitsuru Murata $\cdot$ Haruhito Kikuchi Izumi Takei · Takeo Nakayama · Kiyoaki Watanabe Kazuyuki Omae

\title{
Assessment of tailor-made prevention of atherosclerosis with folic acid supplementation: randomized, double-blind, placebo-controlled trials in each MTHFR C677T genotype
}

Received: 17 January 2005 / Accepted: 17 March 2005 / Published online: 14 May 2005

(C) The Japan Society of Human Genetics and Springer-Verlag 2005

\begin{abstract}
This study aimed at assessing the effect of folic acid supplementation quantitatively in each MTHFR C677T genotype and considered the efficiency of tailormade prevention of atherosclerosis. Study design was genotype-stratified, randomized, double-blind, placebocontrolled trials. The setting was a Japanese company in the chemical industry. Subjects were 203 healthy men after exclusion of those who took folic acid or drugs known to effect folic acid metabolism. Intervention was folic acid $1 \mathrm{mg}$ /day p.o. for 3 months. The primary endpoint was plasma total homocysteine level (tHcy). In all three genotypes, there were significant tHcy decreases. The greatest decrease was in the TT homozygote [6.61 (3.47-9.76) $\mu \mathrm{mol} / 1]$ compared with other genotypes [CC: 2.59 (1.81-3.36), CT: 2.64 (2.16-3.13)], and there was a significant trend between the mutated allele number and the decrease. The tHcy were significantly lowered in all the genotypes, but the amount of the de-
\end{abstract}

K. Miyaki $(\square) \cdot$ K. Omae

Department of Preventive Medicine and Public Health, School of Medicine, Keio University,

35 Shinanomachi, Shinjuku-ku,

Tokyo 160-8582, Japan

E-mail: miyaki@sc.itc.keio.ac.jp

Tel.: + 81-3-33531211

Fax: $+81-3-33593686$

M. Murata $\cdot$ I. Takei

Department of Internal Medicine,

School of Medicine,

Keio University,

Tokyo, Japan

M. Murata $\cdot$ H. Kikuchi $\cdot$ I. Takei $\cdot$ K. Watanabe

Department of Laboratory Medicine,

School of Medicine, Keio University,

Tokyo, Japan

T. Nakayama

Department of Health Informatics,

Kyoto University School of Public Health,

Kyoto, Japan crease differed significantly in each genotype, which was observed at both 1 and 3 months. Using these timeseries data, the largest benefit obtained by the TT homozygote was appraised as 2.4 times compared with the $\mathrm{CC}$ homozygote. Taking into account the high allele frequency of this SNP, this quantitative assessment should be useful when considering tailor-made prevention of atherosclerosis with folic acid.

Keywords Arteriosclerosis · Homocysteine $\cdot$ Folic acid - Dietary supplements - Methylenetetrahydrofolate reductase $(\mathrm{NADPH} 2) \cdot$ Polymorphism

\section{Introduction}

In 1969, McCully reported the vascular pathology of severe, inherited, homocysteinemia for the first time (McCully 1969). Since then, many studies on hyperhomocysteinemia have been conducted, and elevated plasma total homocysteine (tHyc) level has become an established risk factor for atherosclerotic vascular diseases in many persuasive systematic reviews (Boushey et al. 1995; Welch and Loscalzo 1998; Hankey and Eikelboom 1999). Investigations at a genetic level have also progressed and have revealed not only considerably rare mutations that cause severe inherited homocysteinemia but also common polymorphisms, which moderately elevate the homocysteine level. The latter has attracted particular attention in the field of public health because of its high allele frequency in the general population.

In the metabolic pathway of homocysteine, 5,10methylenetetrahydrofolate reductase (MTHFR, EC 1.5.1.20) is one of the key enzymes, as well as methionine synthase and cystathionine beta synthase. In the genes of these enzymes, some single nucleotide polymorphisms (SNPs) have been identified, and many studies are elu- 
cidating the profiles of these polymorphisms. Among these SNPs, a common $\mathrm{C}$ to $\mathrm{T}$ substitution at position 677 of the MTHFR gene (MTHFR C677T polymorphism) results in an alanine to valine amino acid substitution in the protein and leads to a $30 \%$ decrease of enzyme activity in the heterozygote and a $60 \%$ decrease in the homozygote (Frosst et al. 1995). This homozygote (TT genotype) was known to cause mild hyperhomocysteinemia more frequently than the major allele homozygote. Furthermore, its allele frequency approaches $30 \%$ in many ethnic groups (Wilcken 2003). Thus, in the present study, we focused our attention upon this SNP as the target for tailor-made prevention of atherosclerosis.

There was uncertainty over whether the association between MTHFR C677T polymorphism and atherosclerotic diseases actually existed. In 1998, Brattstrom et al. reported a negative result in a meta-analysis of the relative risk of vascular disease associated with the TT genotype $1.12(0.92-1.37)$ (Brattstrom et al. 1998). In 2002, however, this hypothesis came to be positively supported by two persuasive meta-analyses (Klerk et al. 2002; Wald et al. 2002) with more data included. [Klerk et al. reported CHD risk as $1.16(1.05-1.28)$, and Wald et al. 1.21 (1.06-1.39).] Thus, it is inferred that the MTHFR C677T TT genotype is an independent risk factor for atherosclerosis via mild hyperhomocysteinemia. In addition, B vitamin supplementation such as folic acid has been shown to lower homocysteine levels (Homocysteine Lowering Trialists' Collaboration 1998; Vermeulen et al. 2000; Wald et al. 2001). The MTHFR C677T homozygote could therefore be a target of effective prevention by folic acid supplementation from the viewpoint of high-risk approach. However, quantitative data do not currently exist regarding the degree of response to folic acid in each genotype. This study thus aimed at assessing the effect of folic acid supplementation quantitatively in each MTHFR C677T genotype and considered the efficiency of tailor-made prevention of atherosclerosis in genotype-stratified, randomized, double-blind, placebo-controlled trials prepared in concordance with the CONSORT statement (Moher et al. 2001).

\section{Materials and methods}

\section{Study subjects}

Since we intended to examine the effect of folic acid supplementation as the primary prevention against atherosclerosis, we needed to recruit healthy people. Considering the higher incidence and mortality of atherosclerotic diseases in men than women, we focused on healthy men only. After the approval of the ethical committee of Keio University School of Medicine, we obtained the written informed consent from 210 healthy men for participation in the study. They were considered genetically unrelated according to the information by the company. Subjects in this trial finally consisted of 203 healthy men after exclusion of those who were taking folic acid or drugs known to effect folic acid metabolism. Mean age and body mass index (BMI) were $45.8 \pm 11.5$ years and $23.7 \pm 3.66 \mathrm{~kg} / \mathrm{m}^{2}$ (mean $\pm \mathrm{SD}$ ), which are typical values in Japanese healthy male workers. The follow-up period was from December 2003 to March 2004. The flow diagram of the progress throughout the phases of randomized trials is shown in Fig. 1.

\section{Study design}

The study was designed around genotype-stratified, randomized, double-blind, placebo-controlled trials (nested RCTs). Intervention consisted of folic acid $1 \mathrm{mg} /$ day per o.s. or an identical-looking placebo for 3 months since tHcy reduction is known to be maximal at a folic acid dosage of $1 \mathrm{mg} /$ day (a minimum of $0.8 \mathrm{mg} /$ day has been reported as necessary to achieve the maximum reduction in the tHcy level) (Vermeulen et al. 2000). The primary endpoint was the change in the plasma tHcy level, and the secondary endpoint was changes in the pulse wave velocity (PWV), ankle-brachial pressure index (ABI), and high-sensitive C-reactive protein (hsCRP) levels. All the measurements were done at baseline, 1, and 3 months after the start of intervention. As for deciding when intervention should be stopped in any subject, we made arrangements for monitoring and judging unexpected adverse events, including vitamin-B12-deficiency anemia, the masking of which is the only known side effect of folic acid supplementation. We decided that if any important adverse events judged by doctors in our group were observed, we would stop the trial quickly, and vitamin B12 levels were continuously monitored in all subjects to prevent vitamin-B12-deficiency anemia.

To generate the random allocation sequence, we used SAMPSIZE ver.2 software (Blackwell Science, London, UK) on a Windows workstation RX-65 (Sony, Tokyo, Japan). Randomization (blocking size set as four) was done after genetic stratification of three genotypes (i.e., independent three times randomization). Subjects were randomly allocated to the folic acid group and placebo group at a ratio of one to one. The doctor and nurses in the clinic had no information of the group assignment, and the nurses measuring PWV/ABI and the blood chemistry technicians also had no information of group assignment or any subject profiles. The success of blinding was evaluated by comparing the follow-up rate and the mean capsule-intake rate between both groups.

\section{Measurements}

Age and smoking status were self-reported, and medical history was acquired by interview. Height, weight, systolic and diastolic blood pressures, fasting blood glucose 
level, serum lipid levels [total cholesterol, triglyceride, high-density lipoprotein (HDL) cholesterol], aspartate aminotransferase (AST), alanine aminotransferase (ALT), gamma-glutamyl transpeptidase (gamma-GTP), blood urea nitrogen (BUN), serum creatinine $(\mathrm{Cr})$, and serum uric acid (UA) were measured in all subjects.

To monitor the intake of the folic acid or placebo capsules, we asked all participants to report the actual daily intake every month in a specific format and measured the serum folate level at baseline, 1 , and 3 months after the start of intervention. We also monitored the vitamin B12 level at the same time to prevent vitaminB12-deficiency anemia. Serum folate and vitamin B12 concentrations were measured using chemiluminescence enzyme immunoassay (CLEIA) on UniCel DxI 800 immunoassay systems (Beckman Coulter Inc., CA, USA).

Fasting blood samples were drawn, and plasma for tHcy determination was separated with minimal delay and stored at $-20^{\circ} \mathrm{C}$ until analysis. thcy levels were assayed using the high-performance liquid chromatography (HPLC) method (Ubbink et al. 1991). The PWV and ABI were measured with the AT-form PWV/ABI (Nippon Colin, Aichi, Japan), which can monitor

Fig. 1 Process during phases of the randomized trials bilateral brachial and ankle pressure wave forms simultaneously using the volume plethysmographic method, with two optional tonometry sensors for carotid and femoral arterial wave measurements. The hsCRP was measured using the N-latex CRP II kit (Dade Behring Inc., IL, USA).

Genotyping for the MTHFR C677T polymorphism was performed by the polymerase chain reaction (PCR) technique and restriction fragment length polymorphism (RFLP) analysis. We designed PCR primers 5'CCCAGCCACTCACTGTTTTAG-3' (sense) and 5'TGGGAAGAACTCAGCGAACT-3' (antisense) with DNASIS Pro Ver.2.0 (Hitachi Software Engineering Co. Ltd., Tokyo, Japan). Since the $\mathrm{C}$ to $\mathrm{T}$ transition at nucleotide 677 produces a Hinf I digestion site, the amplified 469-bp product derived from the mutant gene was cleaved into 393-bp and 76-bp fragments by Hinf I (TaKaRa Bio Inc., Shiga, Japan), which leaves the wildtype gene unaffected. After electrophoresis through $6 \%$ polyacrylamide gel, the digestion products were visualized by staining with ethidium bromide.

\section{Statistics}

We calculated the sample size according to the results of our pilot trial. We estimated the standard effect size in

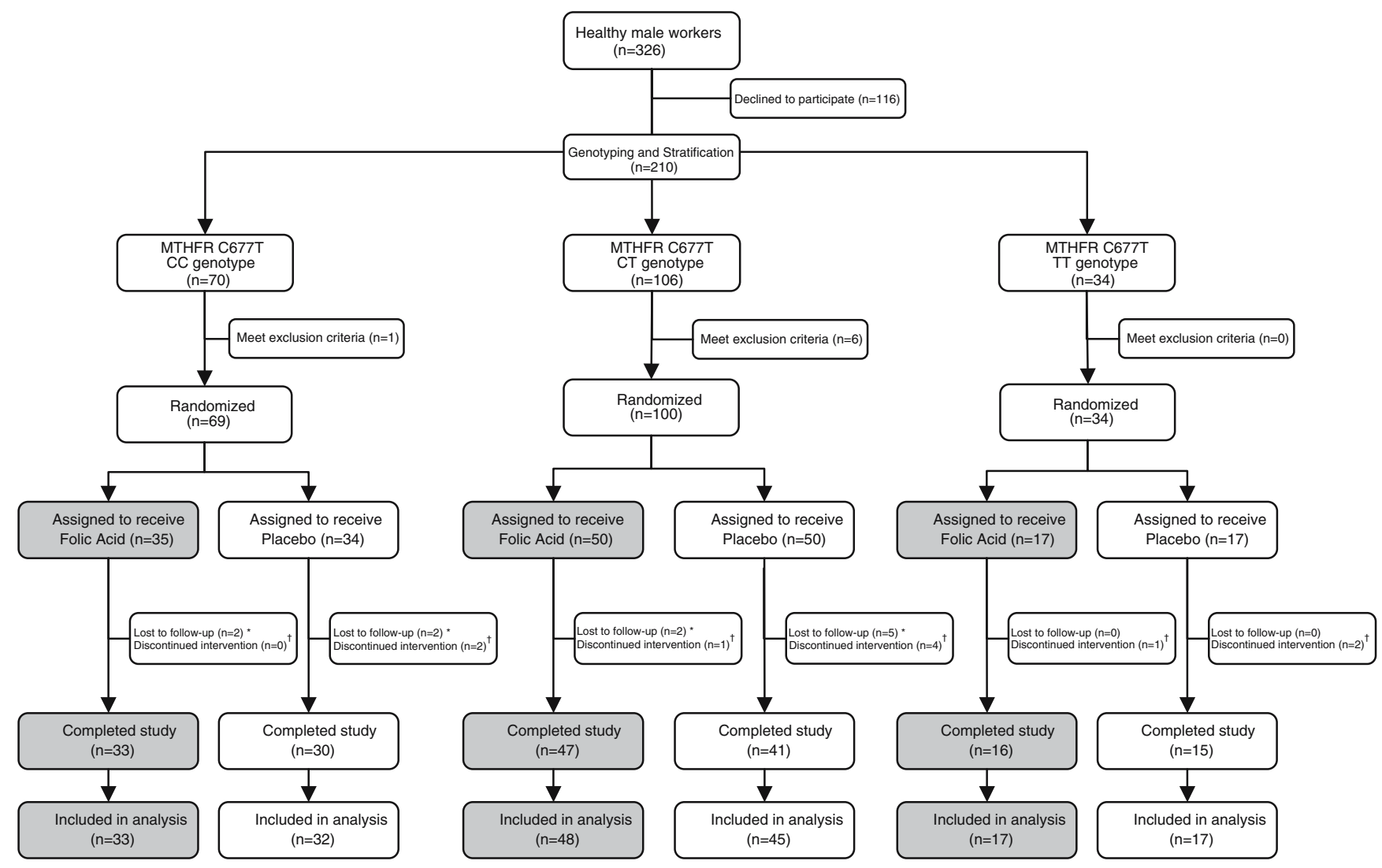

Overall follow up rate after 3 months : $94.6 \%$ (CC : 92.8\%, CT : 93.0\%, TT : 100\%)

* No post randomization contact

† Discontinued intervention means insufficient mean capsule intake rate $(<70 \%)$ 
each genotype (CC, CT, and TT) as $0.5,0.55$, and 0.75 by the serum folate stratified analysis of the cross-sectional data in the pilot study. The planned enrollment of 68,56 , and 32 in each genotype gave an $80 \%$ power to detect a pairwise difference, with a two-sided test at $\alpha=0.05$.

Analysis was based on the intention-to-treat policy. The distributions of triglyceride, HDL cholesterol, AST, ALT, $\gamma$-GTP, Cr, folate, vitamin B12, tHcy, and hsCRP concentrations were all skewed and regarded as log-normal. Therefore, these data were logarithmically transformed and Student's $t$ test was applied. As to age, since the distribution was skewed and not lognormal, Wilcoxon's rank sum test was applied. The other variables were regarded as normally distributed and Student's $t$ test was applied. As to cross-table analysis, the chi-square test or Fisher's exact test were applied. Hardy-Weinberg equilibrium was also assessed by the chi-square test. Comparisons with the baseline level were conducted with a paired $t$ test. Differences in the above variables among different genotype subgroups (multiple comparisons) were assessed with $\mathrm{Tu}-$ key's post hoc analysis of variance (ANOVA). Odds ratios (OR) and 95\% confidence intervals (95\% CIs) were calculated using logistic regression analysis. Changes were evaluated from the baseline level in each group. All statistical analyses were performed using SPSS for Windows version 11.0 (Statistical Product and Service Solutions, IL, USA), and statistical significance was accepted for a two-tailed $P<0.05$. Interim analysis was done at 1 month after the start of intervention.

\section{Role of the funding source}

The sponsor of this investigator-initiated project (the Ministry of Economy, Trade and Industry, Japan) had no role in study design; in collection, analysis, or interpretation of data; in writing the paper; or in the decision to submit the paper for publication.

\section{Results}

Genotyping for the MTHFR C677T polymorphism in the 210 healthy Japanese male subjects showed that 70 were homozygous for the major allele (CC genotype), 106 were heterozygous (CT genotype), and 34 were homozygous for the minor allele (TT genotype). These results were in Hardy-Weinberg equilibrium and consistent with the results previously reported in another Japanese population (Nishio et al. 1996; Lwin et al. 2002). Seven the subjects (CC 6; CT 1; TT 0) who were taking folic acid or drugs known to effect folic acid metabolism were excluded.

The baseline characteristics of the study subjects in each of the MTHFR C677T genotypes (CC, CT and TT) in the folic acid and placebo groups are shown in Table 1 .

Of the 203 people who were randomly allocated into the trial groups, 11 (folic acid group four, placebo group seven) were lost during the follow-up. Two subjects complained of a chilled sensation in the hands or numbness in the fingers and stopped taking the capsules. Both were in the folic acid group. Of the remaining 192 people, ten (folic acid group two, placebo group eight) discontinued intervention (less than $70 \%$ mean capsule intake rate), and 182 completed the study. Thus, the intention-to-treat population consisted of 192 people, and the follow-up rates in the folic acid and placebo groups were $96.1 \%$ and $93.1 \%$, respectively. The flow of participants through each stage is shown in Fig. 1. Reviewing the records on the capsule intake revealed that the mean capsule-intake compliance rates were $91.8 \%$ and $92.8 \%$ in the folic acid and placebo groups, respectively.

As shown in Fig. 2, the serum folic acid level in the folic acid group was significantly higher than in the placebo group at both 1 and 3 months after the start of intervention (both $P<0.001$ ). The plasma tHcy level in the folic acid group was significantly lower than in the placebo group at both 1 and 3 months (both $P<0.001$ ).

There were no significant differences between the folic acid and placebo groups in regard to hsCRP, PWV, and ABI after the intervention. There were no significant differences between groups in regard to changes from the baseline level in hsCRP, PWV, and ABI. These are the results of the intervention trial without considering genotypes.

In addition to the significant increase of folic acid and significant decrease of tHcy, there were also significant changes in the folic acid groups in all three genotypes. As shown in Table 2, the TT homozygote group showed the largest decrease in tHcy, and there was a significant linear trend between the minor allele number and the degree of tHcy decrease. The PWV decreased in folic acid group at 1 month, but the trend with the allele number was not observed, and no significant change was observed at 3 months. The hsCRP significantly decreased in the folic acid group at 3 months, but no trend was observed in the allele number. The ABI did not change significantly in either group. In each of the MTHFR C677T genotypes (CC, CT, and TT), there were significant differences between the folic acid and placebo groups in regard to the decrease of plasma tHcy and the increase of serum folic acid, but there were no significant differences between the two groups in regard to the changes in hsCRP, PWV, and ABI after the intervention.

The significant differences between the MTHFR C677T genotypes in the decrease of plasma tHcy in the folic acid group are shown in Fig. 3. The TT homozygote group showed the largest decrease in plasma tHcy at both 1 and 3 months after intervention. Based on the stable results observed twice in the time course, the effect size of tHcy reduction in the TT homozygote subjects 
Table 1 Baseline characteristics in each MTHFR C677T genotype. Mean $(S D)$ or geometric mean $(G S D)$ are shown. AST/GOT aspartate aminotransferase/glutamic oxaloacetic transaminase, $A L T / G P T$ alanine aminotransferase/glutamic pyruvic transami- nase, $\gamma-G T P$ gamma-glutamyl transpeptidase, $h s C R P$ high-sensitive C-reactive protein, $b a P W V$ brachial-ankle pulse wave velocity, $A B I$ ankle-brachial pressure index

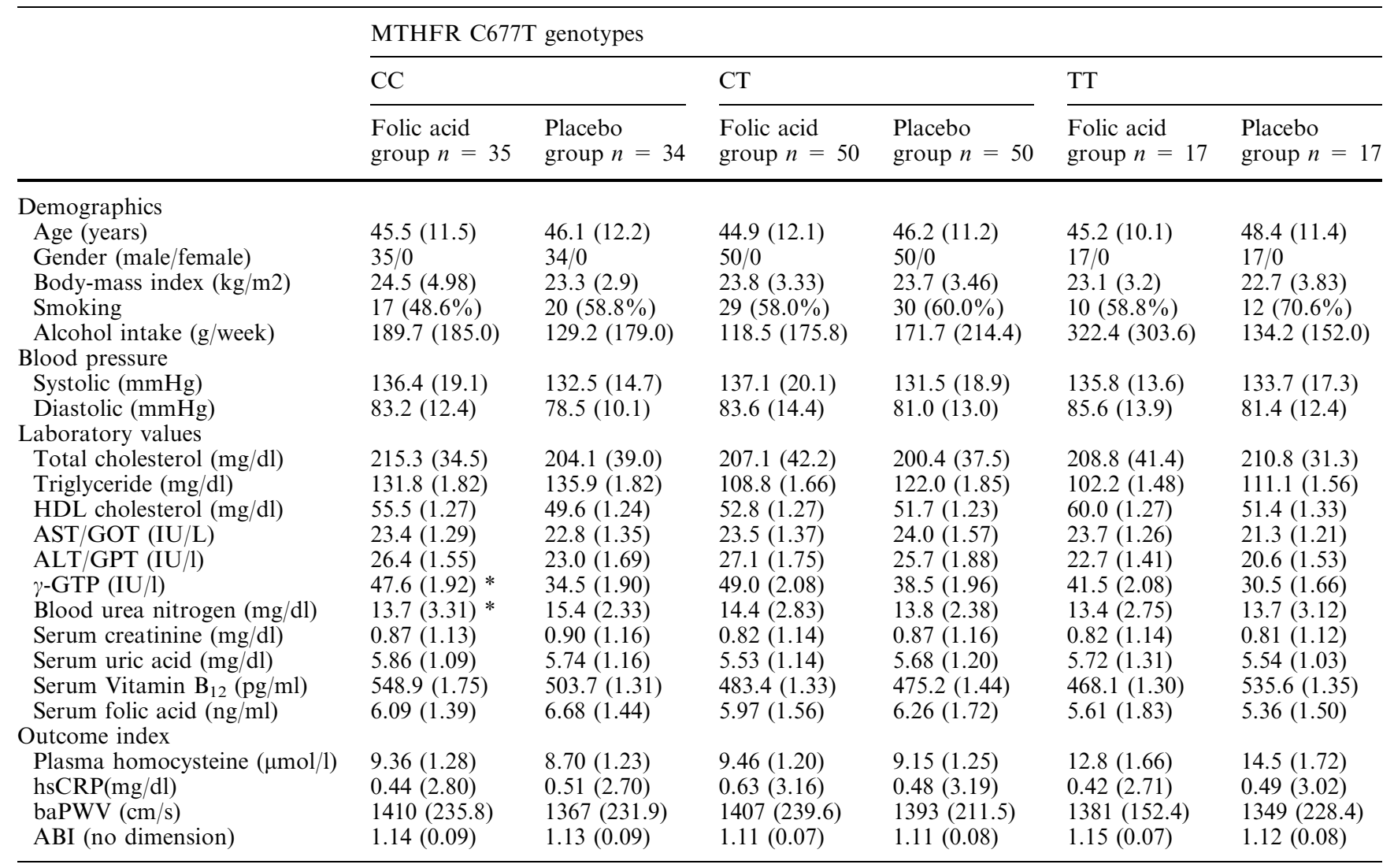

$* P<0.05$ by $t$ test for comparison with the placebo group

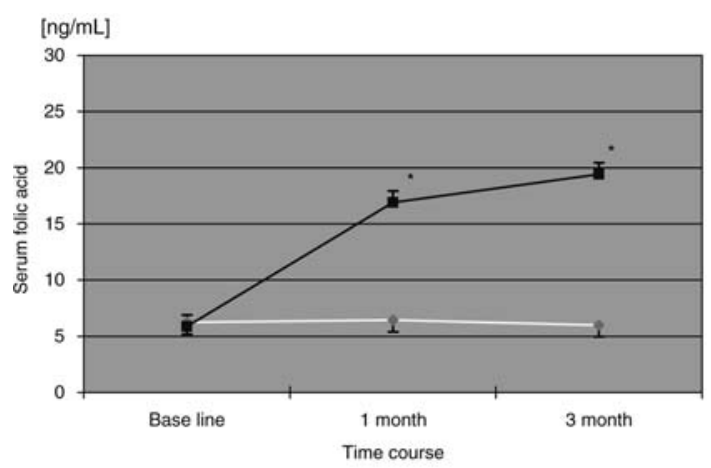

* $p<0.001$ for the comparison with the placebo group. Mean and S.E.M. are shown.

Fig. 2 Serum folic acid and plasma total homocysteine (tHcy) levels in folic acid and control groups

was estimated as $2.4 \times$ compared with the wild type. There were significant linear trends between the allele number and the decrease of plasma tHcy at 1 and 3 months (both $P<0.01$ ). Both these results of the trend tests were statistically significant, even after being adjusted for age, BMI, smoking status, and alcohol
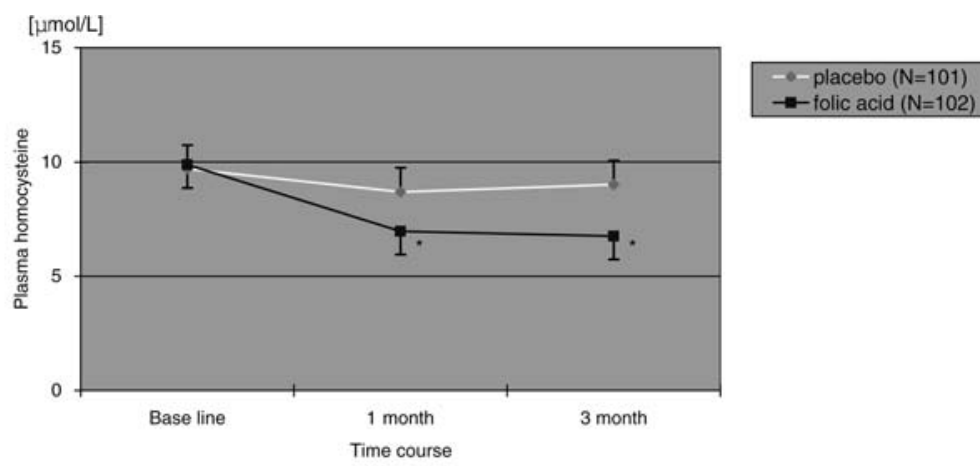
$\rightarrow$ - folic acid ( $N=102$

consumption at 1 and 3 months (both $P<0.01$ ). Tukey's post hoc test of ANOVA demonstrated a statistically larger decrease in the TT genotype group than seen in the CC and CT genotype groups (both $P<0.01$ at 1 and 3 months).

All of the analytical strategies were prespecified, including subgroup analyses and adjustment (no ancillary analysis), and based on the intention-to-treat analysis. 
Table 2 Changes of outcome variables in each MTHFR C677T genotype

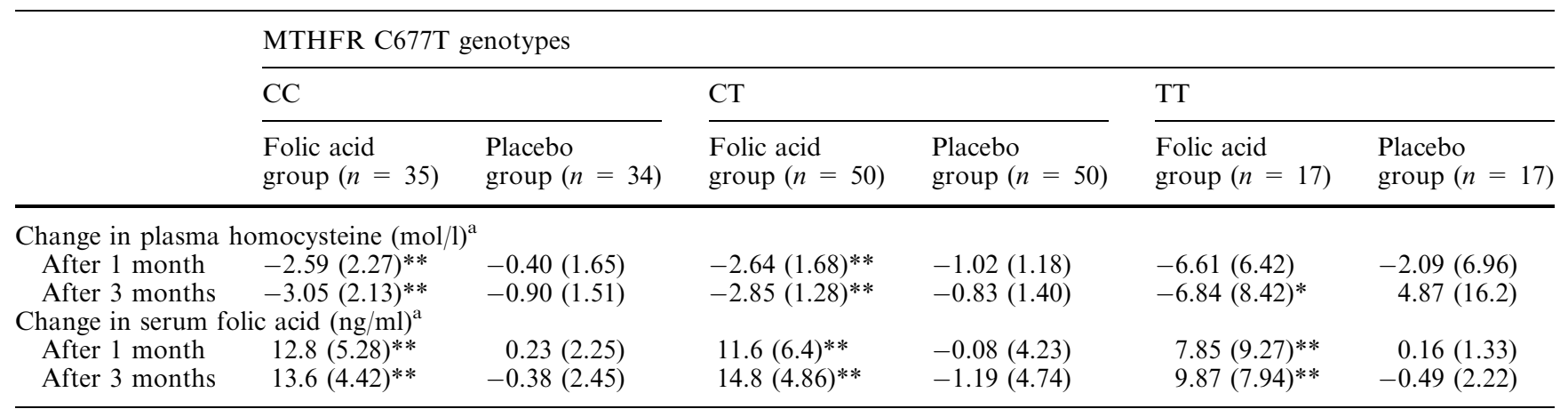

${ }^{\text {a }}$ Mean $(S D)$ is indicated

$* P<0.05 ; * * P<0.01$ for $t$ test versus placebo group. Changes are evaluated from the baseline level in each group
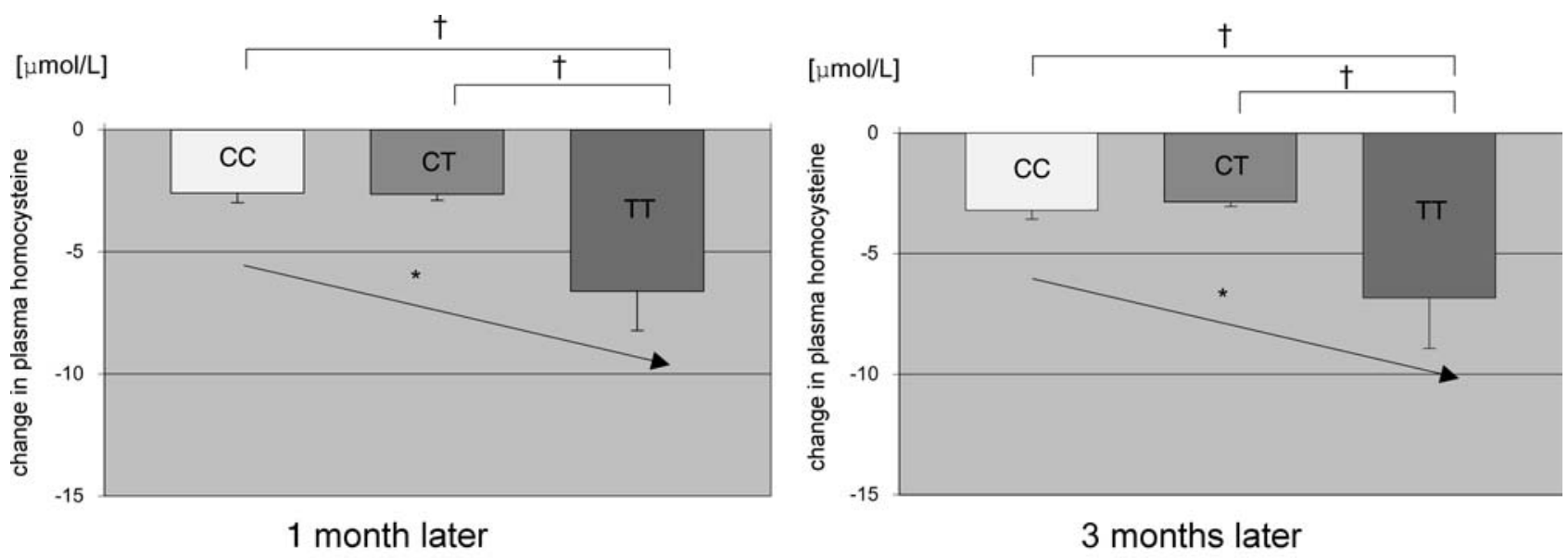

\footnotetext{
Only folic acid group is shown. (Mean and SEM) stand for $p<0.01$ for trend test

These are significant even after being adjusted with age, BMI, smoking status, and alcohol consumption.

${ }^{\dagger}$ stand for $\mathrm{p}<0.01$ for Tukey's post-hoc test of analysis of variance
}

Fig. 3 Differences between MTHFR C677T genotypes in the decrease of plasma total homocysteine (tHcy) in the folic acid group

\section{Discussion}

We have shown that a significant lowering effect of tHcy was observed in the folic acid group in all the MTHFR C677T genotypes, and the degrees of lowering from the baseline level were almost the same at 1 and 3 months after the start of intervention. This indicates that tHcy lowering effect by folic acid supplementation $1 \mathrm{mg} /$ day begins to plateau after 1 month or so. The main result of our study is that the effect size of tHcy reduction in the minor allele homozygote (TT genotype) is estimated to be $2.4 \times$ compared with that in the major allele homozygote. Since there are significant differences in baseline homocysteine levels between the TT genotype and the others, it is possible the baseline level per se influences the degree of change after the intervention. But the greater decrease of tHcy in the TT genotype was not due to the higher baseline level. The change was still larger in the TT genotype, even if the difference of baseline was concerned $(52.5 \%$ reduction in the TT versus $30.1 \%$ in the CC and $29.0 \%$ in the CT genotype). This observation suggests that not only the folate level but also the MTHFR genotype per se influences the responsiveness to folate supplementation. Since this polymorphism is thought to change the affinity of MTHFR to folate (Guenther et al. 1999), this speculation seems reasonable from the viewpoint of molecular mechanisms.

In addition to the fact that the MTHFR C677T polymorphism is very common in many ethnic groups [allele frequency is about $0.30-0.35$ (Wilcken et al. 2003)], it is more important that the mild hyperhomocysteinemia caused by this polymorphism can be improved with folic acid supplementation. Folic acid intake can easily be fortified by trying to take more green vegetables or taking supplements. Venn et al. proved the natural folate intake $(350 \mu \mathrm{g}$ folate derived from food) significantly changed the serum folate status 
in a randomized controlled trial in free-living subjects (Venn et al. 2002). This interaction between the MTHFR genotype and folic acid on the tHcy level is a typical gene-environmental interaction. Thus, this polymorphism is one of the ideal targets of tailor-made prevention from the viewpoint of a high-risk approach.

Since hyperhomocysteinemia is suspected as being a risk factor for many diseases (oral clefts, Down syndrome, placenta-mediated diseases, colorectal neoplasias, Alzheimer's disease, etc.) in addition to vascular diseases and neural-tube defects (Ray and Laskin 1999; Ames 1999; Botto and Yang 2000; Ueland et al. 2001; Ames et al. 2002; Quadri et al. 2004), this information is useful not only for the prevention of atherosclerosis but also for that of other diseases when considering a tailormade preventative approach.

As for our secondary endpoints, we could not find clear results on PWV and ABI in 3 months of folic acid supplementation. There was a significant difference in the PWV at 1 month, demonstrated by the paired $t$ test compared with the baseline in the folic acid group $(P=$ 0.021 ) but not at 3 months. The paired $t$ test showed a significant difference in the ABI at 3 months compared with the baseline in the folic acid group $(P=0.015)$ but not at 1 month. Furthermore, there were no significant differences between the folic acid and placebo groups in regard to changes in PWV and $\mathrm{ABI}$ after the intervention.

Since the PWV and ABI are not fine indices of atherosclerosis and vascular stenosis respectively, to detect any change in such a short period seems difficult. It is compatible with the previous report by Mangoni et al. (Mangoni et al. 2002) that 4 weeks of folic acid intervention $(5 \mathrm{mg} / \mathrm{day})$ did not change the PWV significantly in healthy cigarette smokers. It is also compatible with the previous report by Vermeulen et al. that 2 years of folic acid intervention $(5 \mathrm{mg}$ folic acid $+250 \mathrm{mg}$ vitamin B6/day) did not change the ABI significantly in healthy siblings of patients with premature atherothrombotic disease (Vermeulen et al. 2000). Via lowering the tHcy (Homocysteine Lowering Trialists' Collaboration 1998; Vermeulen et al. 2000; Wald et al. 2001) or directly improving endovascular dysfunction (Mangoni et al. 2002), folic acid may improve the PWV and ABI, but 3 months does not seem long enough to detect any significant changes. Longer observation will be needed to clarify this association in Japanese.

Although Doshi et al. reported that folic acid reduces intracellular endothelial superoxide (Doshi et al. 2001) and Nakano et al. reported folic acid protects against oxidative modification of human LDL (Nakano et al. 2001), we could not find any clear results with folic acid supplementation on hsCRP. Although there was a significant decrease in the hsCRP level in the folic acid group at 3 months (paired $t$ test, $P=0.041$ ) compared with the baseline level, the changes from baseline in the folic acid group and those in the placebo group were not different significantly (data not shown).
As has been pointed out by Folsom et al., the B vitamin status is not presumed to be a strong correlate of circulating levels of inflammatory markers (Folsom et al. 2003).

As for generalizability (external validity), these findings were based on the results of a study of healthy Japanese male workers. Whereas the allele frequency of this polymorphism is not so different among ethnic groups, the response to folic acid can be to some extent different. In addition, the baseline level of folic acid will be a more important factor. Dividing our data into the two groups using baseline folate levels (more or less than the median value), the baseline homocysteine levels were significantly different between the TT and CC genotypes only in the lower folate group but not in the higher folate group. So, it is possible the baseline level of folic acid influences the individual response to folate supplementation. The Food and Drug Administration in the United States mandated the fortification of cereal-grain products with folic acid to prevent neural-tube defects in 1996 (effective by January 1998) (US Food and Drug Administration 1996). According to the report of Jacques et al. (Jacques et al. 1999), plasma folate level changed from 4.6 to $10.0 \mathrm{ng} / \mathrm{ml}$ before and after the fortification (No B vitamin supplements users) using the data of the Framingham Offspring Study Cohort. The baseline level of our study subjects was $6.09 \mathrm{ng} / \mathrm{ml}$ (Table 1). Although such a national intervention is rather rare in the world, the difference in the baseline level of folic acid is very important, as is shown by significant heterogeneity between the results obtained in European populations compared with North American populations in the meta-analysis by Klerk et al.(Klerk et al. 2002).

As for side effects from folic acid supplementation, nothing remarkable was observed. In the dropouts from the folic acid group, one complained of a chilled sensation in the hands and one had numbness in the fingers. Since these complaints were not reported as far as we know, the association is unclear. None of the subjects showed any sign of vitamin B12 deficiency during the serum vitamin B12 monitoring. This finding has verified that oral folic acid supplementation is quite safe, at least for 3 months or so.

In conclusion, we report that the MTHFR C677T minor allele homozygote obtained a 2.4-fold beneficial decrease in thcy levels compared with the major allele homozygote. Considering the high allele frequency of this polymorphism, the results of this quantitative assessment should be useful when considering tailormade prevention of atherosclerosis with folic acid.

Acknowledgements This research was supported by the Ministry of Economy, Trade and Industry, Japan (R\&D for practical use of university-based technology by matching government and private funds). We thank all staff and study participants. Koichi Miyaki conceived the study, designed the protocol, enrolled participants, participated in data collection and analysis, and drafted the manuscript. Mitsuru Murata was responsible for organization of the 
genetic analyses, participated in data collection, and verified all results. Haruhito Kikuchi, Izumi Takei, and Kiyoaki Watanabe advised on important intellectual content for the concept of the study. Takeo Nakayama gave much valuable advice regarding study design. Kazuyuki Omae was responsible for the study design and organization, data interpretation, and overview of the project. All authors were involved in the data interpretation and contributed to the writing of the paper. The first author (Koichi Miyaki) had full access to all the data in the study and takes responsibility for the integrity of the data and the accuracy of the data analysis. No conflicts of interest exists, for any author.

\section{References}

Ames BN (1999) Cancer prevention and diet: help from single nucleotide polymorphisms. Proc Natl Acad Sci USA 96:1221612218

Ames BN, Elson-Schwab I, Silver EA (2002) High-dose vitamin therapy stimulates variant enzymes with decreased coenzyme binding affinity (increased $\mathrm{K}(\mathrm{m})$ ): relevance to genetic disease and polymorphisms. Am J Clin Nutr 75:616-658

Botto LD, Yang Q (2000) 5,10-Methylenetetrahydrofolate reductase gene variants and congenital anomalies: a HuGE review. Am J Epidemiol 151:862-877

Boushey CJ, Beresford SA, Omenn GS, Motulsky AG (1995) A quantitative assessment of plasma homocysteine as a risk factor for vascular disease. Probable benefits of increasing folic acid intakes. JAMA 274:1049-1057

Brattstrom L, Wilcken DE, Ohrvik J, Brudin L (1998) Common methylenetetrahydrofolate reductase gene mutation leads to hyperhomocysteinemia but not to vascular disease: the result of a meta-analysis. Circulation 98:2520-2526

Doshi SN, McDowell IF, Moat SJ, Lang D, Newcombe RG, Kredan MB, Lewis MJ, Goodfellow J (2001) Folate improves endothelial function in coronary artery disease: an effect mediated by reduction of intracellular superoxide? Arterioscler Thromb Vasc Biol 21:1196-1202

Folsom AR, Desvarieux M, Nieto FJ, Boland LL, Ballantyne CM, Chambless LE (2003) B vitamin status and inflammatory markers. Atherosclerosis 169:169-174

Food and Drug Administration, HHS (1996) Food standards: amendment of standards of identity for enriched grain products to require addition of folic acid. Fed Regist 61:8781-8797

Frosst P, Blom HJ, Milos R, Goyette P, Sheppard CA, Matthews RG, Boers GJ, den Heijer M, Kluijtmans LA, van den Heuvel LP (1995) A candidate genetic risk factor for vascular disease: a common mutation in methylenetetrahydrofolate reductase. Nat Genet 10:111-113

Guenther BD, Sheppard CA, Tran P, Rozen R, Matthews RG, Ludwig ML (1999) The structure and properties of methylenetetrahydrofolate reductase from Escherichia coli suggest how folate ameliorates human hyperhomocysteinemia. Nat Struct Biol 6:359-365

Hankey GJ, Eikelboom JW (1999) Homocysteine and vascular disease. Lancet 354:407-413

Homocysteine Lowering Trialists' Collaboration (1998) Lowering blood homocysteine with folic acid based supplements: metaanalysis of randomized trials. BMJ 316:894-898

Jacques PF, Selhub J, Bostom AG, Wilson PW, Rosenberg IH (1999) The effect of folic acid fortification on plasma folate and total homocysteine concentrations. N Engl J Med 340:14491454

Klerk M, Verhoef P, Clarke R, Blom HJ, Kok FJ, Schouten EG, MTHFR Studies Collaboration Group (2002) MTHFR $677 \mathrm{C} \rightarrow \mathrm{T}$ polymorphism and risk of coronary heart disease: a meta-analysis. JAMA 288:2023-2031
Lwin H, Yokoyama T, Date C, Yoshiike N, Kokubo Y, Tanaka H (2002) Are the associations between life-style related factors and plasma total homocysteine concentration different according to polymorphism of 5,10-methylenetetrahydrofolate reductase gene (C677T MTHFR)? A cross-sectional study in a Japanese rural population. J Epidemiol 12:126-135

Mangoni AA, Sherwood RA, Swift CG, Jackson SH (2002) Folic acid enhances endothelial function and reduces blood pressure in smokers: a randomized controlled trial. J Intern Med 252:497-503

McCully KS (1969) Vascular pathology of homocysteinemia: implications for the pathogenesis of arteriosclerosis. Am J Pathol 56:111-128

Moher D, Schulz KF, Altman DG. (2001) The CONSORT statement: revised recommendations for improving the quality of reports of parallel-group randomized trials. Lancet 357:11911194

Nakano E, Higgins JA, Powers HJ (2001) Folate protects against oxidative modification of human LDL. Br J Nutr 86:637-639

Nishio H, Lee MJ, Fujii M, Kario K, Kayaba K, Shimada K, Matsuo M, Sumino K (1996) A common mutation in methylenetetrahydrofolate reductase gene among the Japanese population. Jpn J Hum Genet 41:247-251

Quadri P, Fragiacomo C, Pezzati R, Zanda E, Forloni G, Tettamanti M, Lucca U (2004) Homocysteine, folate, and vitamin B12 in mild cognitive impairment, Alzheimer disease, and vascular dementia. Am J Clin Nutr 80:114-122

Ray JG, Laskin CA (1999) Folic acid and homocyst(e)ine metabolic defects and the risk of placental abruption, pre-eclampsia and spontaneous pregnancy loss: a systematic review. Placenta 20:519-529

Ubbink JB, Vermaak WJH, Bissbort S (1991) Rapid high-performance liquid chromatographic assay for total homocysteine levels in human serum. J Chromatogr 565:441-446

Ueland PM, Hustad S, Schneede J, Refsum H, Vollset SE (2001) Biological and clinical implications of the MTHFR C677T polymorphism. Trends Pharmacol Sci 22:195-201

Venn BJ, Mann JI, Williams SM, Riddell LJ, Chisholm A, Harper MJ, Aitken W (2002) Dietary counseling to increase natural folate intake: a randomized, placebo-controlled trial in freeliving subjects to assess effects on serum folate and plasma total homocysteine. Am J Clin Nutr 76:758-765

Vermeulen EG, Stehouwer CD, Twisk JW, van den Berg M, de Jong SC, Mackaay AJ, van Campen CM, Visser FC, Jakobs CA, Bulterjis EJ, Rauwerda JA (2000) Effect of homocysteinelowering treatment with folic acid plus vitamin B6 on progression of subclinical atherosclerosis: a randomized, placebocontrolled trial. Lancet 355:517-522

Wald DS, Bishop L, Wald NJ, Law M, Hennessy E, Weir D, McPartlin J, Scott J (2001) Randomized trial of folic acid supplementation and serum homocysteine levels. Arch Intern Med 161:695-700

Wald DS, Law M, Morris JK (2002) Homocysteine and cardiovascular disease: evidence on causality from a meta-analysis. BMJ 325:1202

Welch GN, Loscalzo J (1998) Homocysteine and atherothrombosis. N Engl J Med 338:1042-1050

Wilcken B, Bamforth F, Li Z, Zhu H, Ritvanen A, Renlund M, Stoll C, Alembik Y, Dott B, Czeizel AE, Gelman-Kohan Z, Scarano G, Bianca S, Ettore G, Tenconi R, Bellato S, Scala I, Mutchinick OM, Lopez MA, de Walle H, Hofstra R, Joutchenko L, Kavteladze L, Bermejo E, Martinez-Frias ML, Gallagher M, Erickson JD, Vollset SE, Mastroiacovo P, Andria G, Botto LD (2003) Geographical and ethnic variation of the $677 \mathrm{C}>\mathrm{T}$ allele of 5,10 methylenetetrahydrofolate reductase (MTHFR): findings from over 7000 newborns from 16 areas world wide. J Med Genet 40:619-625 